International Journal of Humanities and Social Sciences

p-ISSN: 1694-2620 | e-ISSN: 1694-2639

Vol. 13, No. 1 (2021), pp. 35-45, CIJHSS

https://doi.org/10.26803/ijhss.13.1.4

Received Jan 05, 2021 | Revised Apr 29, 2021 | Accepted May 7, 2021

\title{
Specific Features of Three Newly Published English Textbooks for Elementary School in Japan - A Quantitative Study of the Textbooks
}

\author{
Kiyomi Watanabe \\ Business Administration Dept, Fukuyama Heisei University, \\ Miyuki-cho, Fukuyama-city, Japan
}

\begin{abstract}
As Japan starts English education at the primary level in 2020, the study looks at three sets of English textbooks for the primary level through quantitative analyses. The objective of the study is to find specific features of each textbooks through quantitative analyses, because quantitative studies are known to be useful objective information which qualitative studies often fail to miss. The vocabulary-size analysis shows the following. First, Crown Jr. textbooks have the largest vocabulary size, second, the Correspondence Analysis shows that Crown Jr. textbooks seem to contain some Jr. high school level grammar, and third, Grade 5 textbooks of Junior Sunshine and New Horizon Elementary focus on the alphabets, singing and chanting, and school life; that Grade 6 textbooks of New Horizon Elementary and Junior Sunshine have materials on international foods and culture, and that both grade 5 and 6 textbooks of Crown Jr. contain more third-person sentences. The study believes these findings would provide useful information for those who are engaged in primary and secondary English education in Japan.
\end{abstract}

Keywords: textbook analysis; EFL English textbook; correspondence analysis

\section{Introduction}

Textbooks used in primary and secondary schools in Japan are required to be approved by the Ministry of Education, Culture, Sports, Science And Technology (MEXT). MEXT sets the standard curricula called the Course of Study for primary and secondary education. The approved textbooks are based on the Course of Study, which is normally revised every 10 years.

MEXT has approved seven sets of English textbooks (grade 5 and grade 6 as one set) for the 2021 school year. Each textbook has unique features while they are all approved by MEXT and are based on the Course of Study. For instants, some focus more on verbal communication while others on grammar. Therefore, it renders a valuable challenge for the researchers of English education in Japan to study these textbooks because studying these textbooks can reveal specific features of each textbook and the features unique to the specific revision of the Course of Study.

\section{English education in Elementary school in Japan}

English education at the primary level in Japan began in 2011 when a subject named Foreign Language Activities was included in the then-newly revised Course of Study. Its name "Activities" refers to subjects which are not included in the regular curriculum, such as Math, Japanese, Music, or PE, and these subjects do not require grading. Foreign Language Activity was taught in 
Grade 5 and 6. It mainly focused on listening and speaking.

In the latest Course of Study, which was revised in 2017, English was included among regular subjects, of which a transitory curriculum was to be implemented in 2018 and the full implementation in 2020. The commentary version of the latest Course of Study states the reasons for making English a regular course (MEXT 2017). It states that particular learning issues were found under the former version regarding pronunciation, spelling, and sentence construction (MEXT p. 7). It further states that since pupils in higher grades are at the stage of developing abstract concepts, more systematized learning measures are recommended (Ibid.)

\section{Significance and Aim}

For this study, the author has selected three sets of English textbooks (grade 5 and grade 6 as one set): Crown Jr. (Crown), Junior Sunshine (Sunshine), and New Horizon Elementary English Course (New Horizon). According to an article that appeared in the December 7, 2020 issue of Japan Education Press paper, New Horizon's share comes on top with 57.6\%, Sunshine has $9.1 \%$ (third) and, Crown with 4.8\% (fifth) among the seven textbooks (Japan Education Press 2020).

The aim of this research paper is to quantitatively study the textbooks to find specific features of each textbook. In order to implement this aim, the following research questions will be considered:

1. Are there any specific features among the three sets of textbooks?

2. Are there any specific differences between grade 5 and grade 6 textbooks?

As a quantitative study, this paper analyzes the textbooks in the following area and with the following analysis measure: or (analytic scale)

1. Vocabulary size: token, type, Type/Token Ratio (TTR)

2. Word frequency: Correspondence Analysis

As stated at the outset, even though all the textbooks follow the curriculum stated in the current Course of Study, each textbook has particular features or specific focal points unique to the textbook. Revealing these features and points, the study believes, would provide useful information to those who are engaged in English education in primary and secondary schools in their textbook selection process.

\section{Previous studies}

There are not many but a few helpful quantitative studies on English textbooks. Aikawa (2009) studied the vocabulary of the elementary and jr. high school English textbooks in Taiwan. He found that the number of tokens increases from elementary to jr. high level. He also points out that the type/token ratio tends to be low. He concludes that the low TTR shows that repetition of the words that appeared in the textbooks is rare. Watanabe, Asai and Abe (2016) compared Thai textbooks and Japanese textbooks. They found that Thai textbooks have more words both in token and type. Watanabe, Asai and Akase (2017) compared Chinese textbooks and Japanese textbooks and found that the Chinese textbooks have more tokens and types, but their readability (difficulty) level is lower than that of Japanese textbooks. They assume the Chinese national curriculum intentionally control the readability level of the textbooks.

There is a statistical analysis tool called Correspondence Analysis (CA) that analyzes texts with word-frequency data. Nakamura \& Sinclair (1995) used CA to study Bank of English (English corpus data) and found features specific to spoken English. Tono (2000) studied writing data of grades 7 through 12 utilizing CA, and found that grade 7 through 9 tend to use noun phrase more and grade 10-12 tend to use verb phrase more. Uenishi, Watanabe and Ozasa (2017) studied English textbooks of Jr. high school and high school and concluded that the results of CA correlated with the qualitative results. Watanabe, Fukui (2018) studied Chinese, Taiwanese, and Thai Jr. high school (grade 7) textbooks with CA and found that these three textbooks had features in the areas of readability and exercise questions. Watanabe, Fukui 
and Hosokawa (2019) analyzed eight national universities' entrance exams with CA, and concluded that the texts used in the textbooks could be categorized into three groups: business, culture, and family. Honda, Asai, Watanabe, and Ozasa (2019) used CA to study 12 Japanese historical fifth-year English textbooks from $19^{\text {th }}$ century. The study found that CA proves to be a capable tool to differentiating quantitatively the features of the textbooks.

\section{Vocabulary size analysis \\ Results and discussion}

In this analysis, the total number of words (token), the number of new words (type), and the type/token ratio (TTR) are produced. Table 1 shows the results. As comparative data, the same categorical data of jr. high school (grades 7-9) is provided in Table 2.

Table 1: Vocabulary size

\begin{tabular}{|c|c|c|c|c|c|c|}
\hline \multirow{2}{*}{ textbook } & \multicolumn{2}{|c|}{ token } & \multicolumn{2}{|c|}{ type } & \multicolumn{2}{|c|}{ T'TR } \\
\hline & & ave. & & ave. & & ave. \\
\hline Crown 5 & 8848 & \multirow{2}{*}{8951} & 880 & \multirow{2}{*}{1031} & 9.94 & \multirow{2}{*}{11.19} \\
\hline Crown 6 & 9054 & & 1183 & & 12.44 & \\
\hline New Horizon 5 & 7770 & \multirow{2}{*}{7866} & 755 & \multirow{2}{*}{804} & 9.7 & \multirow{2}{*}{10.2} \\
\hline New Horizon 6 & 7967 & & 853 & & 10.7 & \\
\hline Sunshine 5 & 6808 & \multirow{2}{*}{8129} & 658 & \multirow{2}{*}{701} & 8.86 & \multirow{2}{*}{8.38} \\
\hline Sunshine 6 & 9445 & & 743 & & 7.9 & \\
\hline
\end{tabular}

As Table 1 shows, as far as token (total vocabulary size) is concerned, Sunshine 6 has the highest number. The number would be ranked fourth in Table 2 (the jr. high school textbook token ranking). In fact, as far as their tokens are concerned, they are not much different from those of the jr. high school books. Among these three textbook sets, in the average of the two books (grade 5 and 6), Crown comes at the top as having 8951 words on average, Sunshine comes next with 8129 words, followed by New Horizon with 7866 words.

Table 2: Vocabulary size of two sets of jr. high school textbook

\begin{tabular}{|l|c|c|c|}
\hline Jr. high textbook & token & type & TTR \\
\hline New Crown 1 & 8504 & 1144 & 13.45 \\
\hline New Crown 2 & 9881 & 1369 & 13.85 \\
\hline New Crown 3 & 9977 & 1605 & 16.09 \\
\hline New Horizon 1 & 7983 & 1001 & 12.54 \\
\hline New Horizon 2 & 8071 & 1402 & 17.37 \\
\hline New Horizon 3 & 9847 & 1598 & 16.23 \\
\hline
\end{tabular}

Table 1 also shows that in the category of type (new words), the highest number belongs to Crown 6 (1183). Its number outscores the rest in the list, as the second number is a mere 880 of Crown 5. However, the type in jr. high schools list (Table 2), even Crown 6 would only come in the $5^{\text {th }}$. Jr. high schools' types on average outnumber those of the elementary schools' textbooks by far, which suggests that there is less repetition of words in jr. high textbooks.

Before going into TTR results, it is necessary to briefly touch upon what TTR would actually tell us. According to J. Read (2016), there are four areas that describe the level of vocabulary (lexical) richness: lexical variation/diversity, lexical sophistication, lexical density, and the number of errors, in which TTR is referred to as the core benchmark for lexical diversity. Read points out that "proficient writers have a larger vocabulary knowledge that allows them to avoid repetition by using synonyms, superordinates, and other kinds of related words" (Read, p. 200). On the other hand, Ishikawa (2008) studies Meunier (2008) and concludes that native speakers' TTR tends to stay low while that of advanced learners of second language tends to be higher (Ishikawa, p. 219). He concludes that lexical diversity is not necessarily a benchmark warranting the texts' quality (ibid.). 
With these understandings in mind, the current study looks into the results shown in Table 1. Crown 6 has the highest ratio of 12.44, and it is followed by New Horizon 6. Both Sunshine 5 and 6 textbooks have a rather lighter ratio of 8.86 and 7.9. However, as Ishikawa (2008) stated above, points out the fact that native speakers tend to have lower TTR, the low ratio of Sunshine textbooks may merely refer to some specific features unique to the textbooks.

\section{Analysis of Word Frequency: Correspondence Analysis Results and Discussion}

Correspondence analysis summarizes a set of data, in this case, the eight exams and the Center test, and produces eight categories based on the word frequency. The categories are named as dimensions. CA is a data reduction procedure similar to factor analysis. It describes the relationships among nominal variables (in this study, 16 exams) while simultaneously describing the relationships between the nominal dimensions for each variable. Its results are often displayed in a two-dimensional graphical map since the first and second dimensions have the highest contribution rate among all of the dimensions. Ishikawa, Maeda, Yamazaki (2010) describes CA as one of the handiest statistical analyses for categorizing textual data (p. 245). Greenacre (2017) states that CA is "particularly helpful in analyzing cross-tabular data in the form of numerical frequencies, and results in an elegant but simple graphical display which permits more rapid interpretation and understanding of the data" (Greenacre, p. xi).

As stated in "Previous Study" earlier in the paper, the author of this study has used CA as a textual analysis tool and is strongly convinced that this statistical measurement tool can visually show how the target texts correlate with each other. In the current study, the word frequency cross-tabulation in which the columns contain the number of frequency and rows with the names of six textbooks. The cross-tabulation table is then readjusted according to "double adjustment protocol" suggested by Fukui and Watanabe (2020) before CA was performed. CA computes the tabulation, weighs its rows and columns to make an orthogonal computation, and produces five dimensions.

Correspondence Analysis produces a list of vocabularies which number's one less than the number of total texts analyzed, which are often called dimension. The current study analyzes six textbooks, hence five dimensions. Each dimension lists the words with a frequency number used in the textbooks. Plus and minus in each dimension list refer to two groups of words which CA extracted as contrasting groups. The words on each side are gathered according to common variables in the 6 textbooks. And studying those words in the lists and the textbook(s) in which the words in the list are more frequently used would suggest features unique to the textbook(s).

Table 3: Correspondence Analysis Results (correlation values)

\begin{tabular}{|l|r|r|r|r|r|}
\hline & \multicolumn{1}{|c|}{$\operatorname{Dim} 1$} & \multicolumn{1}{|c|}{$\operatorname{Dim} 2$} & \multicolumn{1}{|c|}{$\operatorname{Dim} 3$} & \multicolumn{1}{|c|}{$\operatorname{Dim} 4$} & \multicolumn{1}{|c|}{$\operatorname{Dim} 5$} \\
\hline eigen value & 0.149 & 0.106 & 0.089 & 0.059 & 0.053 \\
\hline correlation & 0.387 & 0.326 & 0.298 & 0.243 & 0.231 \\
\hline contribution rate & 0.327 & 0.232 & 0.195 & 0.129 & 0.117 \\
\hline cumulative contribution rate & 0.327 & 0.559 & 0.754 & 0.883 & 1 \\
\hline
\end{tabular}

The results of CA are often depicted on a bi-plot map to graphically display interrelations of cross-tabular results. However, the display is only effective if the cumulative contribution rate of the first and second dimensions is larger than 0.5. The cumulative contribution rate in Table 3 shows that the ratio Dimension 1 and 2 combined is 0.559 , suggesting that Dimension 1 and 2 graphically depict $55.9 \%$ of the association that exists between the variables (Beh \& Lombardo, 2014). Thus, the author of the study believes that mapping the six textbooks based on these two dimension results on a bi-plot map would be statistically meaningful. 
Table 4: Correspondence Analysis Results (6 textbooks)

\begin{tabular}{|l|r|r|r|r|r|}
\hline & \multicolumn{1}{|c|}{ Dim 1 } & \multicolumn{1}{c|}{ Dim 2 } & \multicolumn{1}{c|}{ Dim 3 } & \multicolumn{1}{c|}{$\operatorname{Dim} 4$} & \multicolumn{1}{c|}{$\operatorname{Dim} 5$} \\
\hline Crown 5 & 0.072 & -0.361 & 0.034 & -0.427 & 0.168 \\
\hline Crown 6 & 0.611 & -0.339 & -0.046 & 0.272 & -0.148 \\
\hline New Horizon 5 & -0.3 & 0.047 & -0.456 & 0.18 & 0.251 \\
\hline New Horizon 6 & 0.231 & 0.535 & -0.177 & -0.205 & -0.224 \\
\hline Sunshine 5 & -0.628 & -0.173 & 0.164 & 0.048 & -0.317 \\
\hline Sunshine 6 & 0.042 & 0.28 & 0.502 & 0.138 & 0.236 \\
\hline
\end{tabular}

On Dimension 1 column, Crown 6 has the highest value (0.611) followed by New Horizon 6 (0.231), Crown 5 (0.072), and Sunshine $6(0.042)$. The lowest value on the minus side belongs to Sunshine $5(-0.628)$ followed by New Horizon $5(-0.3)$. Therefore, on Dimension 1, Crown 6, New Horizon 6, Crown 5, and Sunshine 6 constitutes one category while Sunshine 5 and New Horizon 5 makes another category. As for Dimension 2 column, New Horizon 6 has the highest value (0.535), followed by Sunshine $6(0.28)$, and New Horizon 5 (0.047). On the minus side, Crown 5 has the lowest value $(-0.361)$ closely followed by Crown $6(-0.339)$, and Sunshine 5 comes next (-0.173). Therefore, on Dimension 2, New Horizon 6, Crown 5, Crown 6, and Sunshine 6 are grouped in one category and Crown 5, Crown 6, and Sunshine 5 makes another category.

On the bi-plot map of Figure 1, the categories found in Dimension 1 and Dimension 2 are shown graphically on the map. The mapping is done based on the tabulation of Table 4. The horizontal bar on the map represents Dimension 1 results, and the vertical bar with Dimension 2 results, shown in Table 4.

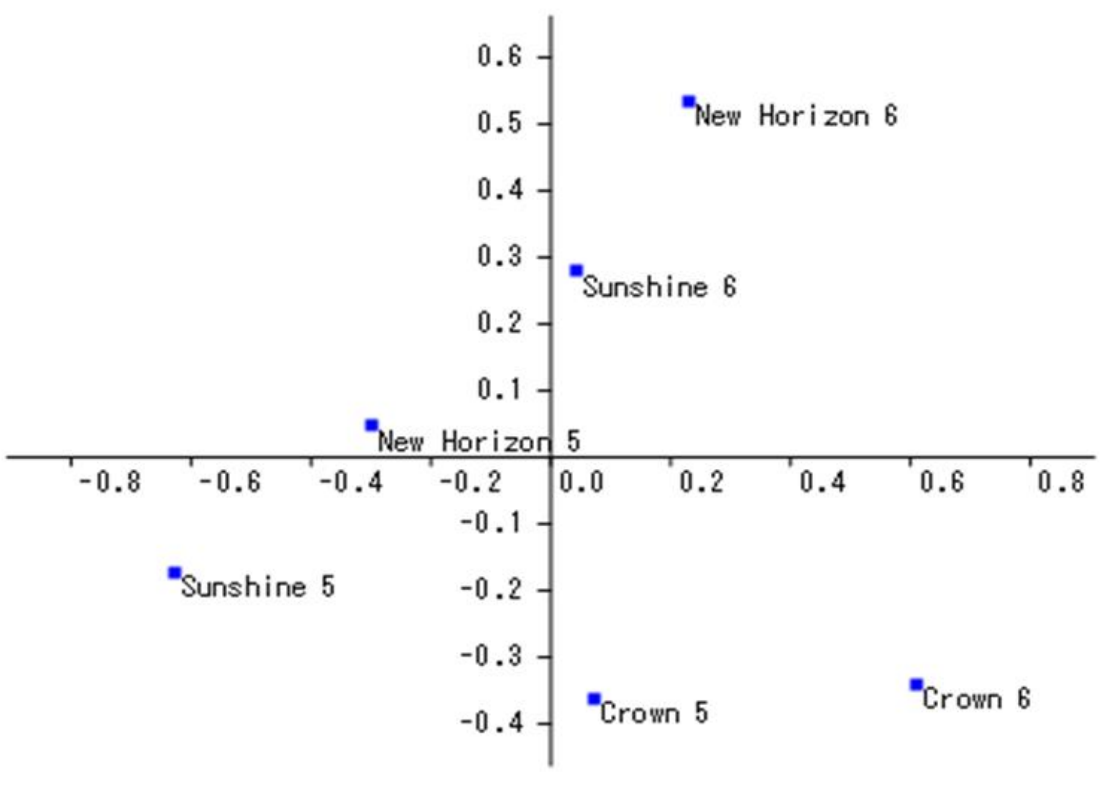

Figure 1: Bi-plot map of Dimension $1 \& 2$

On the map of Figure 1, Sunshine 5 appears on the far left side (minus side) on the horizontal bar distancing itself from the rest of the textbooks, showing that the textbook has unique features of its own. On the vertical bar, New Horizon 6 is placed on the far top side (plus side), and both Crown 5 and Crown 6 appear on the far downside (minus side), which shows that New Horizon 6 has some contrasting features. New Horizon 5 is vertically placed very close to the horizontal bar, suggesting that the textbook may not contain features extracted and contrasted in Dimension 2. 
In order to ascertain the mapping displayed by CA results, the study utilized cluster analysis, which provides the results on a dendrogram. Figure 2 is the dendrogram based on the tabulation in Table 4, showing the statistical distance and interrelations among the six textbooks. It shows that Crown 5 and Crown 6 are placed very close to each other, suggesting that their interrelation is tighter than their relations with the rest of the textbooks. On the left side, New Horizon 5 and Sunshine 5 are placed together, again, showing their tight relationship. New Horizon 6 and Sunshine 6 are placed together in the center of the figure though the above bar is connected to the left, suggesting that they are closer to Crown 5 and 6. It is clear that the bi-plot map and the dendrogram both show very similar mapping: the closeness between Crown 5 and 6, Sunshine 5 and New Horizon 5, and between New Horizon 6 and Sunshine 6.

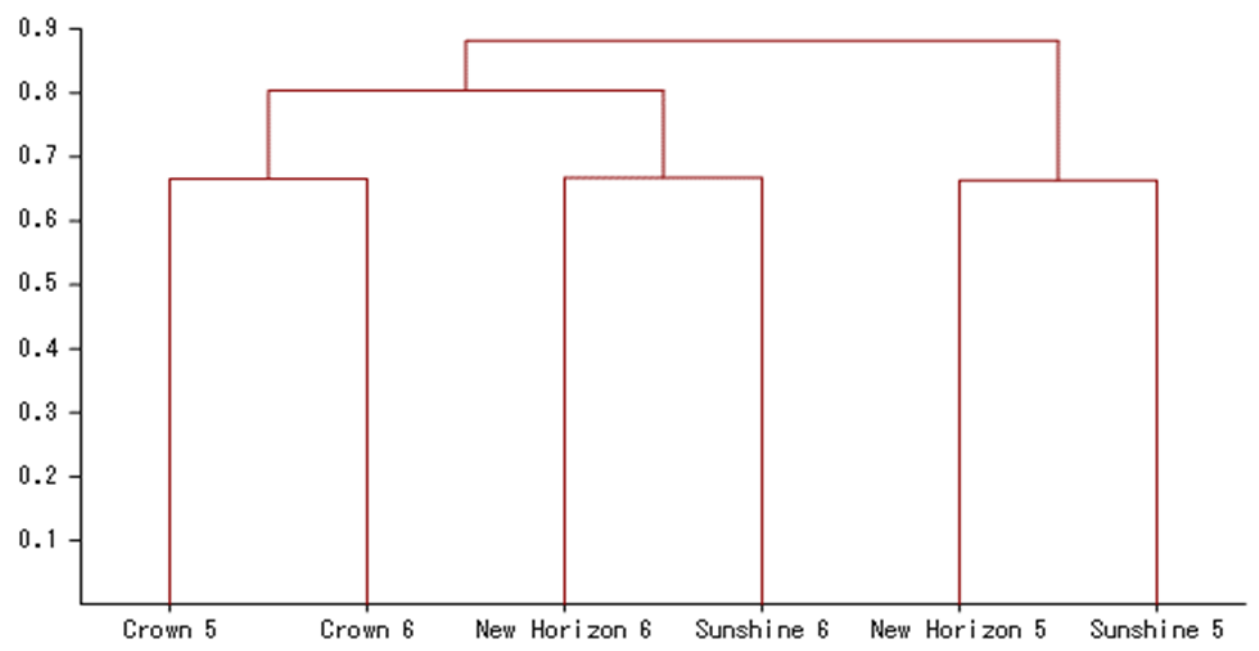

Figure 2: Dendrogram

The following tables (Table 5 - Table 8 ) list the top 20 words of plus and minus sides of Dimension 1 and 2. In the tables, a Dimension column lists the values produced by CR, and columns bearing textbook names provide the frequency of the word in each textbook. By carefully studying these words, the current study tries to find specific features commonly shared by the textbooks in each dimension.

Table 5 is the list of the top 20 words of Dimension 1 plus side. There are many plural nouns and pronouns: "children," "some," "they," "we," "people," and "our." There are also quite a few past tense verbs: "went," "was," "did," "saw," and "ate." The newly revised Course of Study for primary school states regarding the use of pronouns that basic pronouns such as "I," "you," "he," and "she" are to be included in textbooks. However, it does not refer to the rest of the available pronouns, except it states that the grammatical use of pronouns is dealt with at the junior high school level (p. 95). As for past tense verbs, the Course of Study states that it also belongs to the junior high school level (p. 96).

After carefully considered the above findings, the current study suggests that the plus side of Dimension 1 features plural nouns and past tense words, which are to be covered in junior high school. In other words, Crown Jr. 6 contains the words which are expected to be learned at the secondary level.

Table 5: Dimension 1 (plus side) top 20 words

\begin{tabular}{|l|r|r|r|r|r|r|r|}
\hline & Dim 1 & Cr 5 & Cr 6 & N H 5 & N H 6 & Sun 5 & \multicolumn{1}{c|}{ Sun6 } \\
\hline children & 1.208 & 6 & 41 & 3 & 3 & 0 & 0 \\
\hline some & 1.035 & 12 & 47 & 4 & 3 & 0 & 6 \\
\hline they & 1.027 & 13 & 36 & 0 & 0 & 0 & 10 \\
\hline went & 0.861 & 0 & 50 & 0 & 37 & 1 & 30 \\
\hline
\end{tabular}




\begin{tabular}{|l|r|r|r|r|r|r|r|} 
was & 0.832 & 0 & 63 & 0 & 56 & 2 & 37 \\
\hline we & 0.831 & 12 & 234 & 35 & 83 & 8 & 78 \\
\hline there & 0.808 & 15 & 19 & 1 & 11 & 0 & 1 \\
\hline did & 0.797 & 0 & 13 & 0 & 45 & 0 & 0 \\
\hline people & 0.712 & 6 & 21 & 4 & 16 & 1 & 5 \\
\hline boy & 0.702 & 92 & 70 & 1 & 0 & 2 & 9 \\
\hline our & 0.697 & 4 & 34 & 12 & 33 & 1 & 1 \\
\hline saw & 0.682 & 0 & 23 & 0 & 15 & 0 & 35 \\
\hline girl & 0.67 & 70 & 56 & 0 & 0 & 4 & 8 \\
\hline now & 0.654 & 15 & 19 & 3 & 5 & 1 & 4 \\
\hline an & 0.633 & 10 & 37 & 8 & 10 & 5 & 4 \\
\hline swimming & 0.627 & 4 & 12 & 1 & 16 & 0 & 15 \\
\hline big & 0.617 & 6 & 32 & 2 & 10 & 7 & 8 \\
\hline person & 0.609 & 0 & 1 & 0 & 69 & 0 & 0 \\
\hline don't & 0.607 & 19 & 17 & 0 & 5 & 2 & 5 \\
\hline ate & 0.599 & 0 & 14 & 0 & 39 & 0 & 29 \\
\hline
\end{tabular}

Table 6 shows the top 20 words in the minus side of Dimension 1, which are contrasted with the plus side of Dimension 1. The textbooks which are placed on this side are Sunshine 5 and New Horizon 5. Thus, the features found from the list would be the features of these two textbooks. The study notices several words that seem to refer to giving directions: "straight," "left," and "turn." The topic of "giving directions" is included as one of the recommended activities in speaking skill in the Course of Study (p. 106). The study also notices alphabet letters: "z," "o," "g," and "y." The Course of Study states that capital and small block letters ate to be dealt with in the textbook (p. 110).

Words referring to singing are also found in the list: "song" and "sing." The Course of Study emphasizes the use of singing and chanting as effective methods for nurturing reading skills (p. 103).

Words relating to subjects taught in school are also found in the list: "math," "swim," and "science." The Course of Study suggests school life as a recommended topic, in which pupils can relate to their everyday activities (pp. 114-115). Speaking of one's birthday or one's heroes are also included in the Course of Study as recommended topics (p. 109). And "birthday" and "hero" appear in the list as well.

After considering the above findings, the study suggests that Sunshine 5 and New Horizon 5 have basic English learning features such as small and capital letters, communicative activities such as giving direction, and self-introduction activity such as talking about one's classes, birthdays, and heroes.

Table 6: Dimension 1 (minus side) top 20 words

\begin{tabular}{|l|r|r|r|r|r|r|r|}
\hline & Dim 1 & Cr 5 & Cr 6 & N H 5 & N H 6 & Sun 5 & Sun 6 \\
\hline straight & -1.286 & 8 & 0 & 29 & 0 & 65 & 0 \\
\hline left & -1.101 & 8 & 0 & 20 & 0 & 26 & 0 \\
\hline song & -1.066 & 1 & 1 & 1 & 2 & 26 & 10 \\
\hline twenty & -1.031 & 0 & 0 & 0 & 1 & 30 & 20 \\
\hline hero & -1.01 & 1 & 1 & 52 & 0 & 22 & 0 \\
\hline sing & -0.863 & 3 & 5 & 21 & 16 & 72 & 31 \\
\hline mr & -0.799 & 14 & 0 & 78 & 0 & 41 & 33 \\
\hline z & -0.799 & 9 & 1 & 3 & 0 & 20 & 12 \\
\hline
\end{tabular}




\begin{tabular}{|l|r|r|r|r|r|r|r|} 
math & -0.789 & 18 & 5 & 14 & 2 & 33 & 4 \\
\hline would & -0.785 & 0 & 7 & 19 & 0 & 15 & 0 \\
\hline swim & -0.782 & 15 & 2 & 22 & 2 & 25 & 6 \\
\hline turn & -0.756 & 11 & 4 & 45 & 14 & 35 & 0 \\
\hline o & -0.749 & 11 & 1 & 6 & 2 & 22 & 12 \\
\hline under & -0.726 & 23 & 0 & 5 & 1 & 19 & 0 \\
\hline science & -0.713 & 10 & 5 & 8 & 7 & 31 & 9 \\
\hline happy & -0.709 & 8 & 11 & 13 & 1 & 29 & 3 \\
\hline for & -0.708 & 13 & 19 & 57 & 24 & 87 & 25 \\
\hline birthday & -0.688 & 8 & 6 & 55 & 22 & 38 & 0 \\
\hline g & -0.682 & 14 & 0 & 1 & 2 & 20 & 13 \\
\hline y & -0.639 & 7 & 2 & 8 & 1 & 14 & 11 \\
\hline
\end{tabular}

Table 7 is the top 20 words list of the plus side of Dimension 2. On the bi-plot map of Figure 1, New Horizon 6 and Sunshine 6 are placed on the plus side on the vertical bar suggesting these two books share some common features. On Table 7, the study noticed that words concerning food and eating are in the list: "curry," "ate," and "eat." After studying the actual texts, the study finds that "favorite" in the list is also used in the context of food. There are a few names of countries in the list: "Japan," "Australia," and "China." Studying the actual text shows that "from" in the list is used in relation to "country." Thus, the study suggests that the plus side of Dimension 2 points to various international cultures and foods. The Course of Study lists reading activities using international tour leaflets to share the foods and cultures among pupils (p. 104). Since New Horizon 6 and Sunshine 6 mainly have these words, the study suggests that international culture and foods are the exclusive features of these two textbooks.

Table 7: Dimension 2 (plus side) top 20 words

\begin{tabular}{|l|r|r|r|r|r|r|r|}
\hline & Dim 2 & Cr 5 & Cr 6 & N H 5 & N H 6 & Sun 5 & Sun 6 \\
\hline person & 1.608 & 0 & 1 & 0 & 69 & 0 & 0 \\
\hline curry & 1.256 & 3 & 2 & 0 & 42 & 1 & 11 \\
\hline did & 1.098 & 0 & 13 & 0 & 45 & 0 & 0 \\
\hline live & 1.062 & 4 & 4 & 21 & 66 & 0 & 19 \\
\hline team & 1.024 & 0 & 0 & 1 & 10 & 0 & 38 \\
\hline memory & 0.964 & 0 & 8 & 0 & 25 & 0 & 32 \\
\hline sea & 0.958 & 8 & 4 & 4 & 51 & 5 & 11 \\
\hline ate & 0.953 & 0 & 14 & 0 & 39 & 0 & 29 \\
\hline write & 0.768 & 7 & 7 & 0 & 32 & 4 & 14 \\
\hline from & 0.752 & 10 & 20 & 31 & 84 & 5 & 4 \\
\hline Japan & 0.74 & 8 & 3 & 22 & 30 & 1 & 59 \\
\hline eat & 0.686 & 52 & 17 & 4 & 115 & 2 & 35 \\
\hline read & 0.675 & 7 & 2 & 5 & 34 & 14 & 25 \\
\hline usually & 0.658 & 13 & 0 & 16 & 27 & 0 & 15 \\
\hline summer & 0.639 & 0 & 12 & 8 & 29 & 9 & 27 \\
\hline Australia & 0.617 & 10 & 2 & 5 & 18 & 0 & 12 \\
\hline enjoyed & 0.617 & 2 & 20 & 0 & 26 & 2 & 41 \\
\hline
\end{tabular}




\begin{tabular}{|l|r|r|r|r|r|r|r|} 
favorite & 0.604 & 1 & 7 & 1 & 6 & 3 & 46 \\
\hline high & 0.58 & 2 & 4 & 6 & 16 & 7 & 15 \\
\hline China & 0.554 & 10 & 2 & 3 & 17 & 2 & 12 \\
\hline
\end{tabular}

Table 8 is the list from the minus side of Dimension 2. Figure 1 shows Crown 5, Crown 6, and Sunshine 5 are placed on the minus side of the vertical bar suggesting these three would share the features depicted in the minus side of Dimension 2. The study notices that the words "boy," "girl," and "children" bear high values. Having studied the content of Crown 5 and Crown 6, the study concludes that these texts use relatively many third person sentences when compared with the rest of the textbooks. The Course of Study sets as one of the goals for speaking skills is the ability to talk about yourself (p. 80), referring to the first-person and the second-person usage. The study believes that because of this particular recommendation, the textbooks have less use of "boy," "girl," and "children," with the exception of the two Crown textbooks. In other words, the study believes from the findings that Crown covers materials which are to be dealt with at the secondary school level.

Table 8: Dimension 2 (minus side) top 20 words

\begin{tabular}{|l|r|r|r|r|r|r|r|}
\hline & Dim 2 & Cr 5 & Cr 6 & N H 5 & N H 6 & Sun 5 & Sun 6 \\
\hline boy & -0.97 & 92 & 70 & 1 & 0 & 2 & 9 \\
\hline girl & -0.953 & 70 & 56 & 0 & 0 & 4 & 8 \\
\hline children & -0.804 & 6 & 41 & 3 & 3 & 0 & 0 \\
\hline am & -0.783 & 35 & 67 & 8 & 3 & 13 & 5 \\
\hline they & -0.748 & 13 & 36 & 0 & 0 & 0 & 10 \\
\hline some & -0.703 & 12 & 47 & 4 & 3 & 0 & 6 \\
\hline under & -0.66 & 23 & 0 & 5 & 1 & 19 & 0 \\
\hline she & -0.645 & 93 & 66 & 45 & 3 & 36 & 11 \\
\hline piano & -0.642 & 21 & 2 & 5 & 0 & 13 & 3 \\
\hline he & -0.619 & 111 & 67 & 60 & 1 & 36 & 17 \\
\hline don't & -0.55 & 19 & 17 & 0 & 5 & 2 & 5 \\
\hline mother & -0.529 & 17 & 13 & 4 & 5 & 4 & 1 \\
\hline now & -0.501 & 15 & 19 & 3 & 5 & 1 & 4 \\
\hline box & -0.469 & 28 & 0 & 1 & 0 & 18 & 15 \\
\hline m & -0.465 & 32 & 21 & 8 & 2 & 30 & 20 \\
\hline happy & -0.448 & 8 & 11 & 13 & 1 & 29 & 3 \\
\hline on & -0.443 & 143 & 35 & 50 & 34 & 63 & 9 \\
\hline math & -0.441 & 18 & 5 & 14 & 2 & 33 & 4 \\
\hline by & -0.421 & 20 & 4 & 8 & 6 & 19 & 0 \\
\hline teacher & -0.406 & 31 & 16 & 7 & 4 & 4 & 16 \\
\hline & & & & & & & \\
\hline
\end{tabular}

Out of the five dimensions Correspondence Analysis has produced, the study selected Dimension 1 and 2 because their cumulative contribution rate of the two dimensions is 0.559 , referring to the fact that the two dimensions would explain almost $56 \%$ of the total analysis result (see Table 3). And from studying the top 20 words in plus and minus sides of the two dimensions, the study finds the following features of the textbooks:

1. According to the Course of Study, Crown 5 and Crown 6 contain more grammatical materials, which are to be dealt with in junior high school: plural pronouns and past 
tense verbs.

2. Sunshine 5 and New Horizon 5 contain sentences related to learning of giving direction, alphabet letters, songs, and names of the subjects learned in primary school, all of which are included in the Course of Study as recommended topics.

3. New Horizon 6 and Sunshine 6 contain many materials in relation to international food and culture, which are also included in the Course of Study as recommended activities.

4. Crown 5 and Crown 6 have more third-person sentences than the rest of the textbooks.

\section{Conclusion}

In order to find answers to the two research questions introduced at the outset, the study implemented two analyses: vocabulary size analysis and Correspondence Analysis. In the vocabulary size analysis, the study found that Crown (5 and 6) contains the largest vocabulary size in that its token (average of the two textbooks) is 8951 and type (average) 1031 (see Table 1). And its TTR figure is also the highest among the three sets of textbooks. Since the other two sets of textbooks (New Horizon and Sunshine) have the same amount of vocabulary, the study suggests that Crown has a larger amount of vocabulary, which eventually requires more learning hours at school.

The results of Correspondence Analysis shows that Crown 6 and New Horizon 6 contains more plural pronouns and more past tense verbs than the rest of the textbooks. In contrast, Sunshine 5 and New Horizon 5 contain more alphabets, more singing materials, and more materials on school activities than the rest of the textbooks. They also contain materials relating to giving directions. It also shows that New Horizon 6 and Sunshine 6 have more materials on international cultures and food. And it also shows that both Crown 5 and 6 have more third-person sentences. The study finds that these unique features found from the CR study match the Course of Study. Thus, the study concludes that all of the six textbooks studied faithfully follow the guidelines suggested in the Course of Study.

The author believes that the findings of the study would render useful information to those who are engaged in teaching English at both primary and secondary schools in Japan because the results of the study were able to present specific features unique to the six textbooks, which are rated as the most popular textbooks by primary schools in Japan.

The author also believes that Correspondence Analysis is again proven to be a useful statistical tool for textual analysis. The author of the study plans to continue to use CA in textual analysis, and by doing so, will try to add necessary changes to make the tool more suitable for textual analyses.

\section{References}

Beh, E. J., \& Lombardo, R. (2014). Correspondence analysis: theory, practice and new strategies. West Sussex: John Wiley \& Sons Ltd.

Fukui, M., \& Watanabe, K. (2020). "Development of a Program Exclusively Used for Text Correspondence Analysis." ICTATLL Japan, (vol. 7, 49-58).

Greenacre, M. (2017). Correspondence Analysis in Practice. Boca Raton, Florida: CRC Press.

Ishikawa, S. (2008). Eigo Corpus to Gengo Kyoiku - Data toshiteno text. Tokyo: Tiashukan.

Honda, R., Asai, T., Watanabe, K., \& Ozasa, T. (2019). "A Correspondence Analysis of Twelve Japanese Historical Fifth-Year English-as-a-Foreign-Language Textbooks." International Journal of Learning, Teaching and Educational Research, 18(12), 79-100. https://doi.org/10.26803/ijter.18.12.6

Ishikawa, S., Maeda, T., \& Yamazaki, M. (2010). Gengo Kenkyu no tame no Toke Nyumon, Kuroshio Shuppan: Tokyo.

Japan Education Press (2020). Article appeared on December 7, 2020 issue.

Meunier, F. (1998). Computer tools for interlanguage analysis: A critical approach. In S. Granger (Ed.). Learner English on computer (pp. 19-37). London: Longman.

Monkasho (2020). Henshu Shuisho. 
Retrieved from https://www.mext.go.jp/a_menu/shotou/kyoukasho/tenji/1416494.htm (Dec. 15, 2020).

Nakamura, J., \& Sinclair, J. (1995). The world of woman in the Bank of English: Internal criteria for the classification of corpora. Literary and Linguistic Computing, 10, 99-110.

Read, J. (2000). Assessing vocabulary. Cambridge University Press. p. 200

Sakai, H., Aida, M., Imai, H., Irie, J., Ota, A., Nagata, E., Kajimoto, A., et al. (2020). Crown Jr. 5. Tokyo: Sanseido.

Sakai, H., Aida, M., Imai, H., Irie, J., Ota, A., Nagata, E., Kajimoto, A., et al. (2020). Crown Jr. 6. Tokyo: Sanseido.

Tamai, A., Ano, K., Hamanaka, K., Abe, K., Arai, K., Arai, H., Iijima, K., et al. (2020). New Horizon Elementary English Course. Tokyo: Tokyo Shoseki.

Tamai, A., Ano, K., Hamanaka, K., Abe, K., Arai, K., Arai, H., Iijima, K., et al. (2020). New Horizon Elementary English Course. Tokyo: Tokyo Shoseki.

The Ministry of Education, Culture, Sports, Science And Technology (MEXT) (2017). Syogakko Gakushu Shido Yoryo Exposit: Gaikkokugo Katsudo • Ggaikokugo. Tokyo: Kairyudo.

Tono, Y. (2000). A corpus-based analysis of interlanguage development: Analyzing part-of-speech tag sequences of EFL learner corpora. In B. Lewardowska-Tomaszezyk \& P. J. Melia (Eds.), PALC 99 Practical applications in language corpora (pp. 323-340). Frankfurt, Germany: Peter Lang.

Uenishi K., Watanabe K., \& Ozasa T. (2017). Correspondence Analysis of Three Japanese EFL Textbooks: In Reference to the Improvement of a Criterion Measure for a Readability Tool. Conference Proceedings, 2017 International Conference on Education, Psychology, and Leaning. August 23-25, Sapporo. 49-60.

Yorozuya, R., Adachi, T., Aritomo, K., Iijima, M., Ito, S., Ujyo, Y., Umemoto, R., et al. (2020). Junior Sunshine 5. Tokyo: Kairyudo.

Yorozuya, R., Adachi, T., Aritomo, K., Iijima, M., Ito, S., Ujyo, Y., Umemoto, R., et al. (2020). Junior Sunshine 6. Tokyo: Kairyudo.

Watanabe K., Fukui M., \& Hosokawa M. (2019). Statistical Characteristics of English Entrance Exams of National Universities in Japan. International Journal of Humanities and Social Sciences, 11(6). 14-27. https://doi.org/10.26803/ijhss.11.6.2

Watanabe, K., Asai, T. \& Abe, N. (2016) . An Analysis of Vocabulary of the Elementary and Jr. high school's English Textbooks in Thailand- in comparison with Japanese English Textbooks. ICTATll (vol. 3, pp. 47-56).

Watanabe, K., Asai, T. \& Akase, M. (2017). A Quantitative Analysis of Vocabulary of the Elementary school English Textbooks in China - In comparison with Japanese English Textbooks. ICTATL Japan (vol. 4, pp. 47-58).

Watanabe, k. \& Fukui, M. (2018). Comparative Study of English Textbooks in Asia. Fukuyama Heisei Daigaku Keieigakubu Kiyo (vol. 14, pp. 85-95). 